\title{
Dysphonia Plica Ventricularis and Emotional Dysregulation
}

\author{
Dr. Edwin Dias ${ }^{1}$, Dr. Mithun S. ${ }^{2 *}$, \& Gouri Lingaraddi ${ }^{3}$ \\ ${ }^{1}$ Professor and HOD, Department of Pediatrics, Srinivas Institute of Medical Science and Research \\ Center, Mangalore, India \\ ${ }^{2}$ Assistant Professor, Department of Psychiatry, Srinivas Institute of Medical Sciences\& \\ Research Centre, Mangalore, India \\ ${ }^{3}$ Intern (MBBS), Srinivas Institute of Medical Sciences\& Research Centre, Mangalore, India \\ *Corresponding Email: mdmitsmj@gmail.com
}

Type of the Paper: Case Report.

Type of Review: Peer Reviewed.

Indexed In: OpenAIRE.

DOI : http://doi.org/10.5281/zenodo.1469971.

Google Scholar Citation: IJHSP

\section{How to Cite this Paper:}

Dias, Ediwn., Mithun S.,\& Lingaraddi, Gouri. (2018). Dysphonia Plica Ventricularis and Emotional Dysregulation. International Journal of Health Sciences and Pharmacy (IJHSP), 2(2), 27-30. DOI: http://doi.org/10.5281/zenodo.1469971.

International Journal of Health Sciences and Pharmacy (IJHSP)

A Refereed International Journal of Srinivas University, India.

(C) With Authors

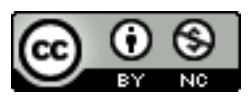

This work is licensed under a Creative Commons Attribution-Non Commercial 4.0 International License subject to proper citation to the publication source of the work.

Disclaimer: The scholarly papers as reviewed and published by the Srinivas Publications (S.P.), India are the views and opinions of their respective authors and are not the views or opinions of the SP. The SP disclaims of any harm or loss caused due to the published content to any party. 


\title{
Dysphonia Plica Ventricularis and Emotional Dysregulation
}

\author{
Dr. Edwin Dias ${ }^{1}$, Dr. Mithun S..$^{2 *}$ \& Gouri Lingaraddi ${ }^{3}$ \\ ${ }^{1}$ Professor and HOD, Department of Pediatrics, Srinivas Institute of Medical Science and Research \\ Centre, Mangalore, India \\ ${ }^{2}$ Assistant Professor, Department of Psychiatry, Srinivas Institute of Medical Sciences\& \\ Research Centre, Mangalore, India \\ ${ }^{3}$ Intern (MBBS), Srinivas Institute of Medical Sciences\& Research Centre, Mangalore, India \\ *Corresponding Email : mdmitsmj@gmail.com
}

\section{ABSTRACT}

Dysphonia plica ventricularis(DPV) is a type of functional dysphonia(FD) which can be caused by several reasons. DPV-emotional type is seen secondary to emotional crisis which is equivalent to psychogenic/dissociative dysphonia. Here we describe a case of DPV secondary to emotional dysregulation with an underlying emotionally unstable personality disorder. Crisis intervention and emphasis on regular compliance to treatment has been discussed.

Keywords: Dysphonia plica ventricularis, Emotionally unstable personality disorder, Dissociation.

\section{INTRODUCTION :}

Dysphonia plica ventricularis(DPV) also known as ventricular dysphoniawas described first in 1935 [1]. Few studies have shown the prevalence of DPV to be around $1 \%$ in the general population and about $4 \%$ of all dysphonias [2], [3]. In DPV dysphonia is seen due to the pathological participation of false vocal folds(FVFs) in phonation which can be habitual, emotional, paralyticetc [4].Emotional type of DPV can be due to an emotional crisis of various reasons [5]. Here we describe a case of DPV emotional type in a 15 year old girl.

\section{CASE REPORT :}

Here we have a 15 year old girl coming from a rural background, studying in $9^{\text {th }}$ standard, residing with her parents and younger brother. She was on regular treatmentfor epilepsy(generalised tonic-clonic type) from past 7 yearswith no seizures reported in past 34 months. She was brought to casualty with an abrupt onset of loss of consciousness of 1 hour duration with no involuntary movements or frothing in the mouth, unlike her previous seizure episodes. On examination her GCS was $9 / 15$, plantar flexor response with no other deficits.Patient was admitted in Intensive Care Unit(ICU) for observation and her blood investigation profile, neuroimaging, chest $\mathrm{x}$ ray done which were within normal limits.
After regaining consciousness while interviewing the child she was responding relevantly by whispering words and in gestures. On clarification parents reported that she used to complain of throat pain from past 1 year but never had such difficulty in talking before. Ear Nose Throat(ENT) reference was sought who found her to be having adenotonsillar hypertrophy with dysphonia plica ventricularis as on laryngoscopy it was found that the child was using FVFs. Elective surgical intervention was advised for enlarged adenotonsilsbut the parents deferred.

On psychiatric evaluation with her parents, she was found to have traits of emotionally unstable personality disorder(EUPD)borderline typenamely emotional instability and threatening self harm. On further probing it was found that intermittently the childgets irritable, skips her meals, has crying spells and verbally abusivetowards family members. Few hours prior to her admission, the child had an altercation with her parents over buying a new mobile phone wherein her parents were punitive with her.Rapport with the child was difficult to establish and hence corroboration of the history with the child was not possible.Before her dysphonia had subsided, the parents requested for discharge thus hampering detailed assessment further. 
A provisional diagnosis of dissociative stupor(F44.2) and dissociative dysphonia(F44.4)along withtraits of EUPD(F60.3.31)was made as per the International Classification of Diseases- $10^{\text {th }}$ revision - clinical descriptions and diagnostic guidelines (ICD-10-CDDG) [6]. Patient was started on Tab. Escitalopram $10 \mathrm{mg}$ and Tab. Lorazepam $1 \mathrm{mg}$ (tapering doses)and advised to continue with the rest of her medications. Brief psychoeducation to the family was given and advised for regular visits but theynever came for follow up.After 2 months the child presented again to the casualty with a similar presentation of loss of consciousness and dysphonia. After relevant interventions and stabilisation of the child, psychiatry reference was given. On clarification with parents no additional information was gained except that in between the 2 admissions she was compliant to medications and her dysphonia intermittently was better. During this admission her dysphonia was seen to be intermittent and serial mental status examinations(MSE's) were done when she was able to verbalise adequately. Child acknowledged the need for help in her emotional dysregulation but denied any other stressors. She was continued on Tab.Escitalopram $10 \mathrm{mg}$ and at the end of 1 week her dysphonia had subsided completely.Psychoeducation to the child and family about her illness was done and behavioural management of her dissociation was explained. For her EUPD she was advised for psychotherapeutic intervention with a clinical psychologist but the parents deferred it. Child was then seen regularly over next 12 months wherein no dysphonia was reported. The main targets on follow-ups were to improve the coping and distress tolerance skills of the child.

\section{DISCUSSION :}

Dysphonia due to psychogenic causes which is a type of FD is included under the dissociative motor disorders as per ICD 10. Dysphonia plica ventricularis(DPV)-emotional type comes under the realm of FD or recently termed as muscle tension dysphonia(MTD) in which dysphonia is seen due to an emotional crisis in absence of any structural or neurological laryngeal pathology [4], [7].
Apart from emotional crisis contributing to functional dysphonia presence of specific personality traits is an important factor in the development and maintenance of FD [6], [7]. Such an emotional crisis can be seen in patients with EUPD as emotional dysregulation is a core feature of this personality disorder [8], thus precipitating dissociation as seen in our case.

Seeking professional help only at times of crisis would render care only to acute management leaving a holistic approach less effective. Hospitalisation(partial) has shown to be more effective than outpatient treatment [9] because patient may be lost for follow ups in outpatient clinics as seen after the first visit in our case. Though for symptomatic relief of affective symptoms antidepressants like selective serotonin reuptake inhibitors(SSRI's) are the choice [10] psychotherapeutic approach like dialectical behavioural therapy forms the mainstay of treatment for personality disorders. Hence the importance of such a psychological intervention needs to be understood by the family.

\section{CONCLUSION:}

DPV can arise secondary to emotional crisis as seen in this 15 year old girl with traits of EUPD. Along with harm reduction strategies long term treatment plans needs has to be put in place to prevent many such episodes in future. Multi-disciplinary team approach, psychoeducation to family, compliance to both pharmacological and non-pharmacological treatments, frequent visits and better insight about the illness in the family form the cornerstones of treatment.

\section{REFERENCES:}

[1] Saunders WH. LIV Dysphonia Plica Ventricularis: An Overlooked Condition Causing Chronic Hoarseness. Ann Otol Rhinol Laryngol. 1956 Sep 1;65(3):66573.

[2] Von Hake CP, Ganzman IP, Mauer TP. Diagnosis and management of ventricular dysphonia. J Am Osteopath Assoc. 1989 Feb;89(2):181-3.

[3] Youssef G. Prevalence, causes andmanagement ofvoicedisordersin Dubai Hospital voice clinic. 2014;18. 
[4] Arnold GE, Pinto S. Ventricular dysphonia: New interpretation of an old observation. The Laryngoscope. 1960;70(12):1608-1627.

[5] Nasri S, Jasleen J, Gerratt BR, Sercarz JA, Wenokur R, Berke GS. Ventricular dysphonia: A case of false vocal fold mucosal traveling wave. Am J Otolaryngol. 1996 Nov;17(6):427-31.

[6] bluebook.pdf (Internet). (cited 2018 Oct 17). Available from: https://www.who.int/classifications/icd/en /bluebook.pdf

[7] Roy N. Functional dysphonia. Curr Opin Otolaryngol Head Neck Surg. 2003 Jun;11(3):144.

[8] Glenn CR, Klonsky ED. Emotion Dysregulation as a Core Feature of Borderline Personality Disorder. J Personal Disord. 2009 Feb 1;23(1):20-8.

[9] Bateman A, Fonagy P. Effectiveness of partial hospitalization in the treatment of borderline personality disorder: a randomized controlled trial. Am J Psychiatry. 1999;156(10):1563-1569.

[10] Mazaira S. (Pharmacological treatment of borderline personality disorder). Vertex B Aires Argent. 2004;15(58):303-8. 